Supporting Information for

\title{
Size-Tailored Porous Spheres of Manganese Oxides for Catalytic Oxidation via Peroxymonosulfate Activation
}

\author{
Qiaoran Liu ${ }^{1}$, Xiaoguang Duan ${ }^{1}$, Hongqi Sun ${ }^{2}$, Yuxian Wang ${ }^{3}$, Moses O. Tade ${ }^{1}$, and \\ Shaobin $\mathrm{Wang}^{1 *}$ \\ ${ }^{1}$ Department of Chemical Engineering, Curtin University, GPO Box U1987, WA6845, \\ ${ }^{2}$ School of Engineering, Edith Cowan University, 270 Joondalup Drive, Joondalup, WA 6027 , \\ ${ }^{3}$ State Key Laboratory of Heavy Oil Processing, China University of Petroleum, Beijing, 18 \\ Fuxue Road, Beijing, 102249, P. R. China
} Australia Australia

\section{*Corresponding authors.}

Email: h.sun@ecu.edu.au (H. Sun); shaobin.wang@curtin.edu.au (S. Wang)

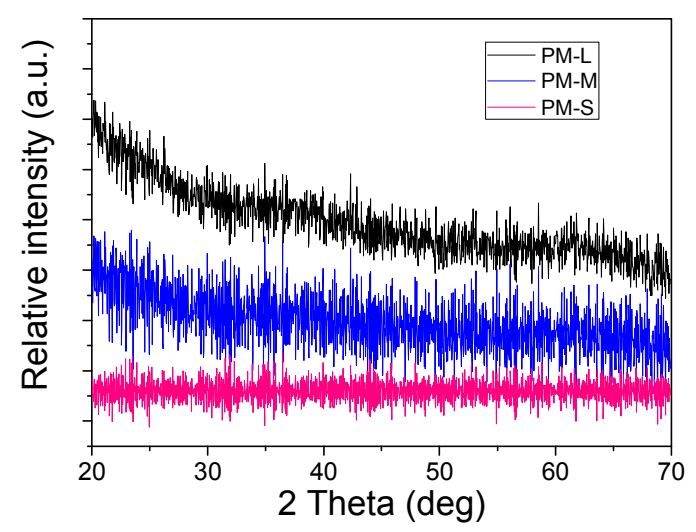

Figure S1. XRD patterns of PM-L (PM-0), PM-M and PM-S obtained with calcination procedures 

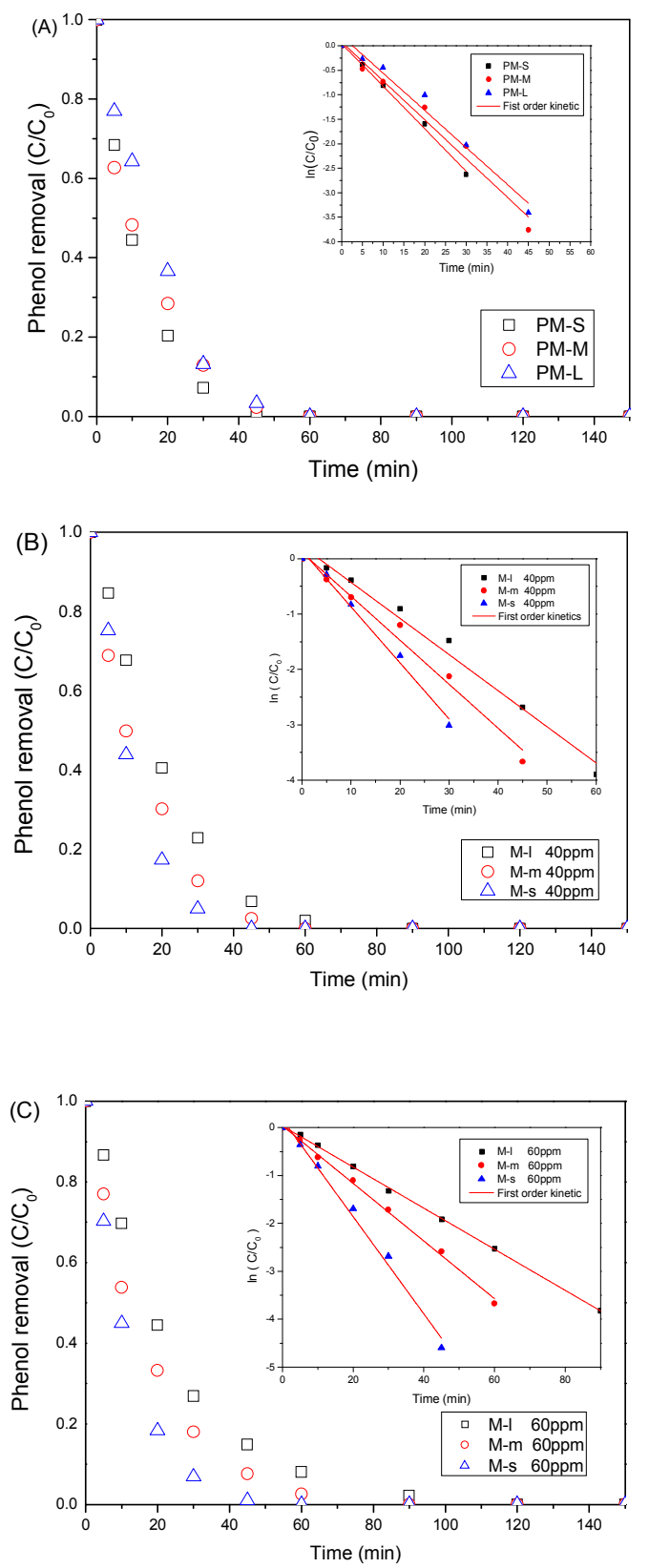

Figure S2. Estimation of rate constants in phenol degradation 

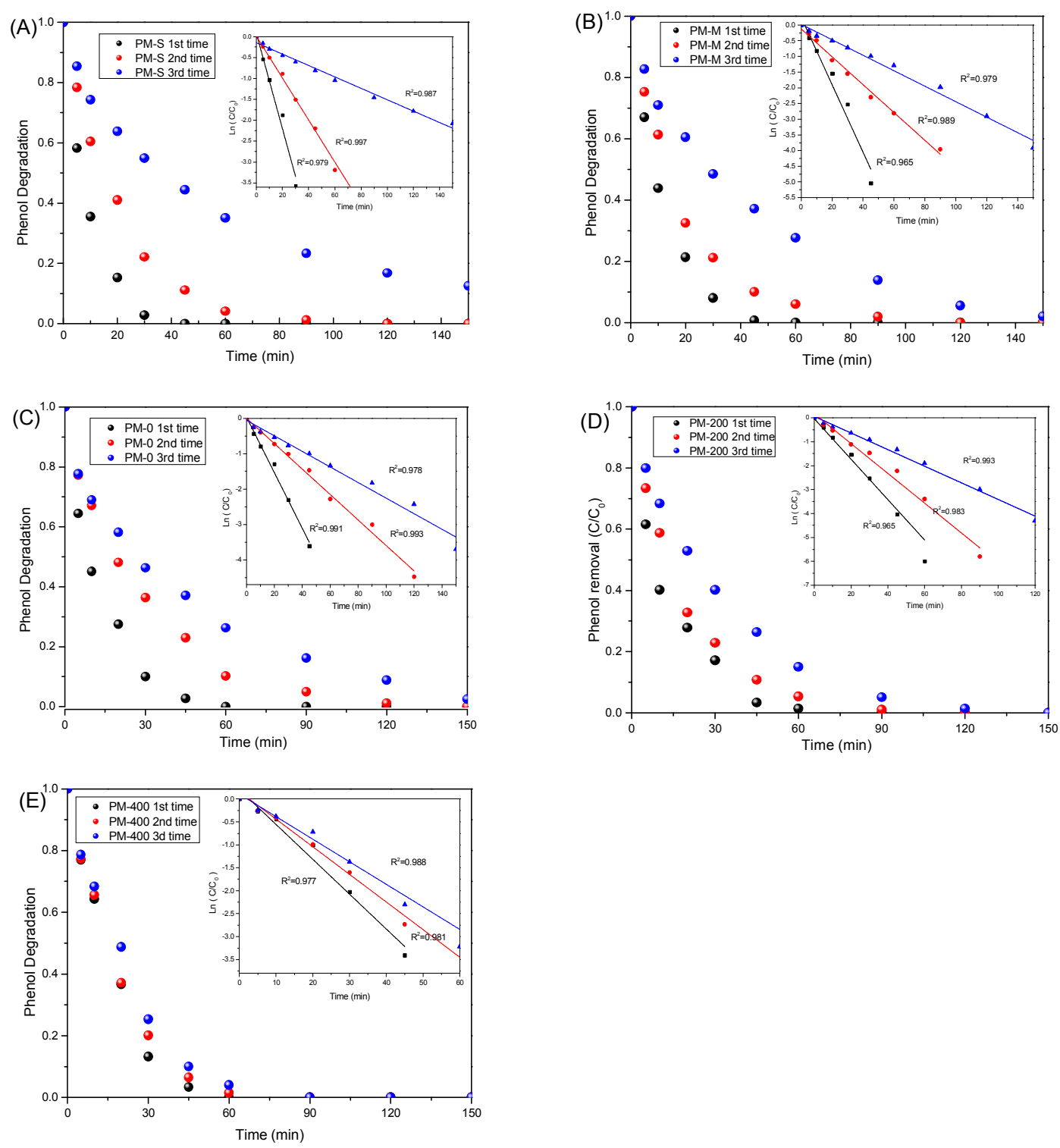

Figure S3. Stability tests of various catalysts 

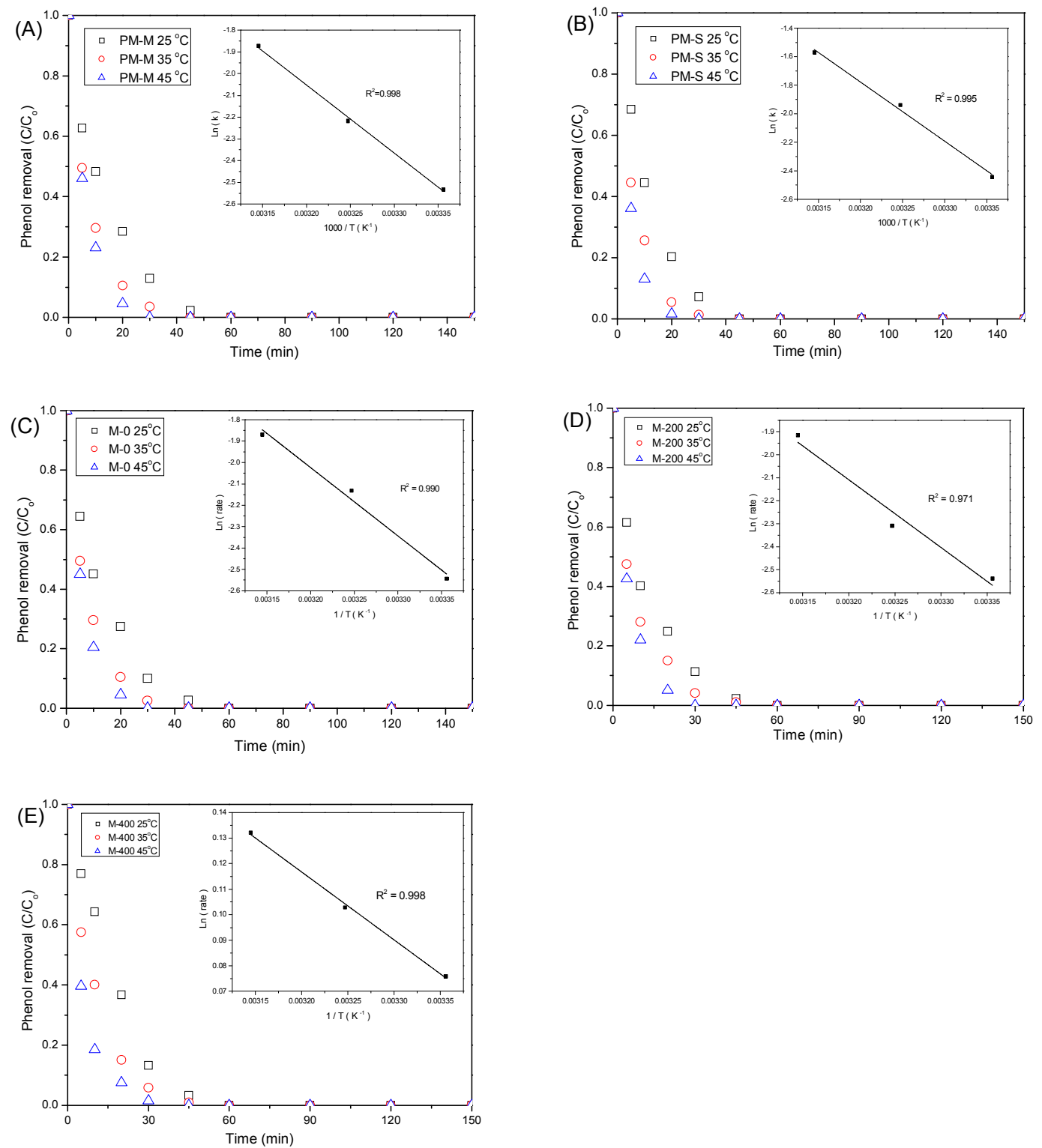

Figure S4. Effect of reaction temperature on degradation and evaluation of activation energy 

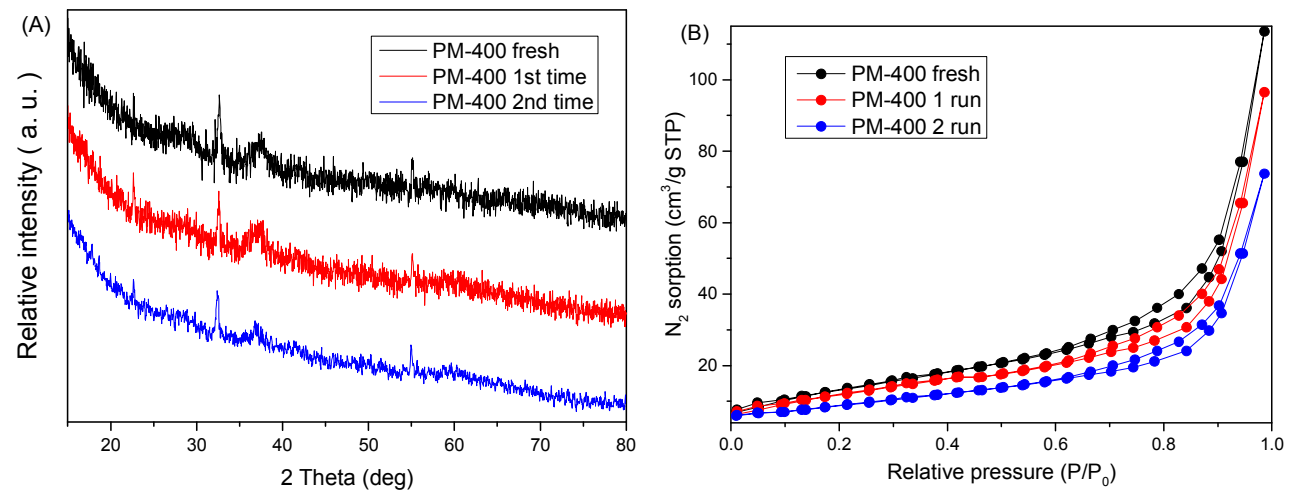

(C)
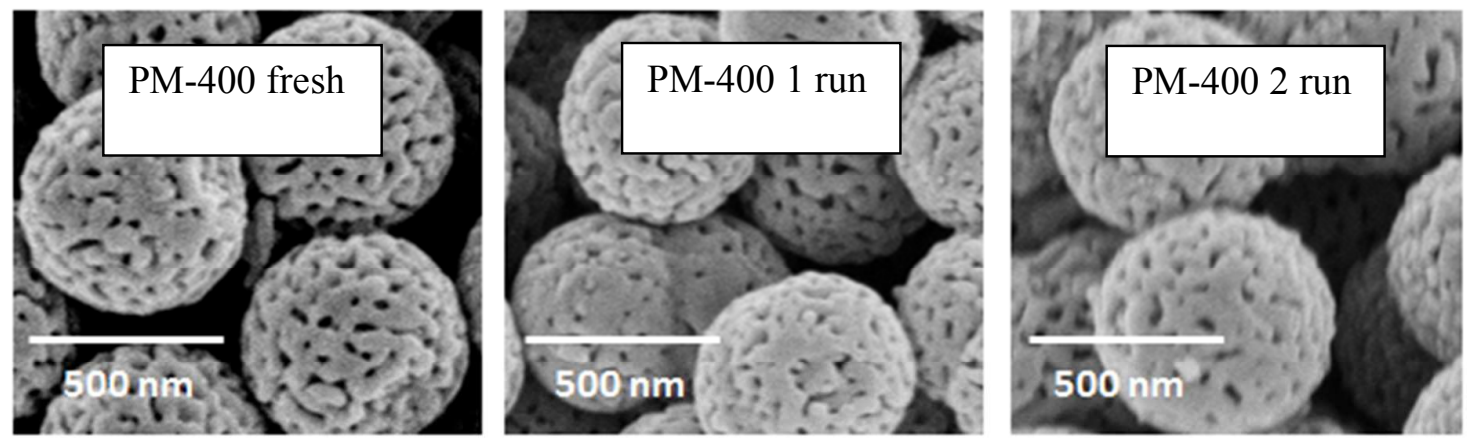

Figure S5. XRD (A), BET (B) and SEM images (C) of PM-400 before and after use. 